\title{
QoS Aware Reliable Cluster based Routing Protocol (QoS-RCBRP) for Heterogeneous Mobile Ad-Hoc Networks
}

\author{
B.Narasimhan \\ Assistant Professor \\ Department of Computer Technology \\ Dr. N. G. P. Arts and Science College \\ (Affiliated to Bharathiar University) \\ Coimbatore -641048
}

\author{
R.Vadivel \\ Assistant Professor \\ Department of Information Technology \\ School of Computer Science and Engineering \\ Bharathiar University \\ Coimbatore - 641046
}

\begin{abstract}
Routing in mobile ad hoc network is an ever demanding research area. The aim of this research work is to design and develop QoS aware reliable cluster based routing protocol (QoS-RCBRP) for heterogeneous mobile ad hoc networks. From the dense number of literatures it has been examined that when the mobility of nodes increases, the performance of several clustering routing protocols gets decreased. Hence the intended routing protocol QoS-RCBRP makes use of mobile backbone to lessen the impact of node mobility. The proposed routing mechanism gets rid of the delay caused by cluster head selection and starts the mobile nodes to initialize the communication instantly after joining the cluster. The QoS metrics packet delivery ratio, delay and throughput are taken into account for comparing the proposed QoS-RCBRP with Ad-hoc On-demand Distance Vector (AODV) routing protocol. From dense simulation results it can be observed that the proposed protocol attains better performance in terms of reduced delay, increased throughput and packet delivery ratio.
\end{abstract}

\section{INTRODUCTION}

Mobile Ad hoc Networks (MANETs) which are wireless networks that are dynamic and self-organizing which allow nodes to establish communication without network infrastructure support. It has been shown by authors of [1] that the network capacity can be improved by deploying backbone nodes which are interconnected by additional high capacity links. The network capacity and routing protocol are the factors impacting scalability. Many routing protocols, like Optimized Link State Routing (OLSR) [2], DestinationSequenced Distance Vector (DSDV) [3], are usually protocols often have scalability issues [4]. For addressing this issue, cluster based routing protocols are proposed to improve the scalability. In cluster based routing, nodes are divided into virtual groups called clusters and there is usually a node called Cluster Head $(\mathrm{CH})$ in each cluster acting as the local controller. All cluster heads together form a network backbone. Topology changes in local cluster can be concealed within the cluster and the local control packets can be contained within each cluster. The control overhead is then reduced and network scalability is improved. The routing process within each cluster is commonly referred to as intracluster routing and routing between clusters is called intercluster routing.

Although several cluster based routing protocols [5-12] have been proposed, the main research focus of cluster based routing has been on the clustering method itself, mainly to achieve different goals such as controlling the cluster size, improving the cluster stability or reducing the latency incurred by the cluster formation process. The problem of clustering has been well studied and many clustering methods have been proposed. Two basic clustering schemes are Lowest ID Clustering [13] and Highest Connectivity Clustering (HCC) [14]. Lowest ID Clustering assumes each node has a node ID and elects nodes with the lowest ID in their neighbourhood as cluster heads. On the other hand, in HCC, clustering nodes with the largest number of neighbors becomes the cluster head. Least Cluster head Change (LCC) [15] improves the cluster stability by restricting the re-clustering condition such that unnecessary re-clustering is avoided. ABP [16] forms clusters based on factors such as node degree and battery level. It attempts to reduce cluster head re-election by introducing a penalty factor such that cluster head is only reelected when the network structure experiences major modification. In [17], the clustering problem is formulated into an Integer Linear Programming (ILP) problem and a heuristic is proposed to generate stable clusters. K-clustering [18-23] is another class of clustering method in which each node is either cluster head or is at most $\mathrm{k}$ hops away from its cluster head. Thus, the size of clusters can be controlled.

\section{RELATED WORKS}

Early MANET routing are broadly classified into two types, proactive or reactive, based on the way they find the routes to destinations. Proactive routing protocols such as Optimized Link State Routing (OLSR) [2] maintain their routing table by periodically exchanging routing information even when there is no data packet to forward. On the other hand, reactive routing protocols such as AODV [32] only find routes to destinations when there are data packets to forward. In general, flat routing protocols often have scalability issues [4]. With large network size, proactive routing protocols could suffer from high control overhead whereas reactive protocols could incur potentially long route discovery delay before data packets can be forwarded. To improve the scalability, cluster based routing is proposed. The basic idea is to restrict the propagation of control packets to reduce the control overhead and isolate local topology changes. The research focus of cluster based routing has been on the clustering method itself since after cluster formation, existing flat routing protocols can be adopted for intra-cluster and intercluster routing. In [11], $\mathrm{Xu}$ and Gerla propose a scheme for heterogeneous MANETs in which powerful nodes with additional backbone links are deployed together with ordinary nodes to form a MANET. This scheme uses Random Competition based Clustering (RCC) to elect cluster heads from these powerful nodes and form K-hop clusters. Any node who does not belong to any cluster can broadcast a packet to announce itself as cluster head and nodes that receive this announcement will 
join this cluster. After cluster formation, AODV and DSDV are used for its inter-cluster and intracluster routing, respectively. This scheme is shown to have better performance compared to the flat AODV. Similarly, Xu et al. propose to adopt LANMAR [46] to support heterogeneous MANET in [10], which is shown to have better delivery ratio and delay performance than flat LANMAR and AODV. Both $[11,10]$ attempt to elect only a subset of the powerful nodes and thus the remaining powerful nodes are underutilized. Theoleyre and Valois [5] take a CDS approach in which some nodes are selected as backbone nodes, the dominators, to form $\mathrm{k}$-CDS backbone. Each node is at most $\mathrm{k}$ hops away from its dominator and knows the topology of its k-hop cluster. Local routes can then be computed. On the other hand, for intercluster routing, it uses a reactive approach similar to AODV. In [6], Niu et al. propose a hybrid cluster based routing protocol (HCR). Unlike many other schemes, it proposes to use reactive routing for intra-cluster routing but adopts a proactive approach for inter-cluster routing. Rasheed et al. [9] proposes a scheme to guarantee efficient intercluster routing by optimizing intermediate cluster selection. The authors propose a cluster ranking mechanism to rank the quality of clusters to facilitate cluster selection. HOLSR $[7,8]$ is another cluster based protocol proposed for heterogeneous MANET. It assumes network nodes are heterogeneous and some nodes may have multiple wireless interfaces. It classifies nodes into different layers of a virtual hierarchy based on their communication capabilities. A node with links to the upper layer of the hierarchy acts as a cluster head in the lower layer. Based on the hop distance to the cluster head which is extracted from the Cluster Id Announcement (CIA) message in HELLO packets, ordinary nodes in the lower layer join the nearest cluster. In each cluster, OLSR [2] is used for intracluster routing. Each node sends out HELLO packets to announce its presence. The HELLO packet contains information about the node itself and the list of addresses of neighboring nodes. HELLO packets are used for neighbor detection and Multipoint Relay (MPR) [33] selection.

Er and Seah [24] proposes a mobility based d-hop clustering scheme MobDHop. This scheme uses signal power to estimate the "closeness" of nodes and in turn derives the related mobility based on the change in this metric. Nodes with lower relative mobility among neighbors will be selected as cluster heads. This scheme allows clusters of similar stability to merge into a single cluster and thus reduces the number of total clusters. In [25] Sakhaee and Jamalipour propose a clustering scheme for pseudolinear highly mobile networks. It makes use of relative velocity information derived from Doppler shift and information obtained from GPS to estimate the relative speed and the expected link time. Nodes with lowest relative mobility with respect to neighboring nodes are elected as cluster head. In [26], authors propose a clustering scheme for networks with group mobility considering not only the mobility but also node degree and power consumption.

Konstantopoulos et al. [28] assumes node's mobility follows certain pattern that can be predicted based on historic information. It proposes to predict the stability of a neighbor by reducing the problem to predicting the next characters in a text given a particular text context has been observed.

Zhang and $\mathrm{Ng}$ [31] proposes a metric called Total Spatial Dependency (TSD) to estimate nodes' mobility, which is computed based on nodes' past speed. The location information is assumed to be made available via GPS. A node with a higher TSD value will have a large neighbor set and similar mobility pattern as its neighbors. Thus nodes with higher TSD values are selected as cluster heads.
In [27], the authors propose to use affinity propagation algorithm which is adapted from data clustering to group nodes with similar mobility pattern together. The simulation result shows it has better reliability than MOBIC. Similar to [25], Ni et al. [29] also proposes to estimate node speed and predict link residual time using Doppler shift. It proposes a Regional Average Relative Speed (RARS) to estimate the relative stability of a node with its neighbors. Nodes with smaller RARS value among neighbors become cluster heads.

Souza et al. [30] proposes another metric called Aggregate Local Mobility (ALM) to estimate the relative mobility of nodes. The computation of ALM is similar to MOBIC but it is based on GPS information instead of signal power of received packets. MPBC [34] is another clustering scheme based on relative node speed. In [35], the clustering is performed based on the vehicle's direction to form stable clusters.

\section{PROPOSED WORK}

This research takes into account of dense heterogeneous networks with high node mobility and low group mobility. There are some nodes in the network which are more powerful than others in terms of communication capability and such nodes are termed as backbone nodes. In the proposed routing scheme, the powerful node is simply based on the configured network interfaces of that node. A node is considered powerful if it is configured with both 802.11 interface and a long range uplink. Information about the network interfaces are assumed to be given through configuration files. Hence, when a node is powered up, it will know whether it has both types of links and can automatically decide its own role without requiring information from other nodes. Once nodes have classified themselves as powerful nodes, they will start acting as cluster heads. It is to be noted that in our scheme, all backbone nodes with both 802.11 and uplinks are used as cluster heads. They do not compete among themselves to elect a set of more powerful nodes as cluster heads. This is to eliminate the cluster head election delay as well as to exploit all available uplink capacity.

During startup, backbone nodes by default become cluster heads. At the time of cluster formation, each cluster head declares its presence by periodically broadcasting ALIVE messages. ALIVE message contains the address of this cluster head and the hop distance to the cluster head dis- $\mathrm{CH}$. The topology of the cluster is embedded into the ALIVE message. Initially dis- $\mathrm{CH}$ is set to 0 and the embedded cluster topology is empty. An ordinary node can join a cluster when receiving a ALIVE message. If multiple ALIVE messages from different cluster heads are received, the node will attach to the cluster head with the smallest hop distance. This hop distance information can be extracted from the received ALIVE message. If a node has the same hop distance to multiple cluster heads, it will choose to join the cluster from which it first receives the ALIVE message. Afterwards, the node will only change to join other cluster if it missed several ALIVE messages from its current $\mathrm{CH}$ or it receives ALIVE messages with smaller hop distance from another cluster head. After joining a cluster, the node will rebroadcast the ALIVE message after incrementing the hop distance field in the message. This allows further nodes to join the cluster. The node will also record the address of the neighbor from which it receives the ALIVE message. A default route Rdft will be added to the routing table using this neighbor as the next hop to the cluster head. It is particularly useful when there is no route available for a destination. In this case the packets can be forwarded to the cluster head. ALIVE messages are emitted 
by the cluster head periodically and each ALIVE is tagged with a sequence number. For each ALIVE message, each cluster member will only relay it once by checking the sequence number. In addition, a node will only relay ALIVE messages received from a node with smaller hop distance than itself to the cluster head. The neighbors' hop distance to the cluster head can be obtained from the received ALIVE message directly. In this way, the ALIVE messages are relayed only outwards towards the cluster boundary. The node is free to leave the current cluster and join another cluster upon receiving ALIVE messages from a new cluster head with smaller hop distance. The node does not need to inform any member of the current cluster. Within each node a lifetime is associated with its cluster head; if a node does not receive ALIVE from its cluster head for a predefined period of time, it will leave the cluster and seek to join a new cluster. ALIVE messages are usually only propagated within each cluster. However, it is possible that two nearby nodes fall into two different clusters. In such scenario, QOS-RCBRP allows nodes at the boundary of clusters to make use of the topology information in ALIVE messages from other clusters so that a more direct and shorter path can be found. The detailed algorithm for cluster formation and maintenance is described in I-Algorithm.

\section{I-Algorithm Cluster Formation and Maintenance}

message msg.

if hasProcessed (seq(msg)) then

drop (msg)

if $(C H(n)==N U L L)_{-}(\operatorname{disch}(n)>\operatorname{disch}(m s g)+1)$ then

$\mathrm{CH}(n)=\mathrm{CH}(\mathrm{msg})$

$\operatorname{disch}(n)=\operatorname{disch}(m s g)+1$

$\operatorname{Rdft}(n)=$ lastHop $(m s g)$

updateRoutingTable()

incrementHopDistance (msg)

rebroadcast (msg)

if $(\mathrm{CH}(n)==\mathrm{CH}(m s g))^{\wedge}(\operatorname{disch}(n)==\operatorname{disch}(m s g)+1)$ then

$\operatorname{Rdft}(n)=$ lastHop $(\mathrm{msg})$

updateRoutingTable()

incrementHopDistance (msg)

rebroadcast (msg)

if $\left.(\mathrm{CH}(n) !=\mathrm{CH}(m s g))^{\wedge} \operatorname{disch}(n)<=\operatorname{disch}(m s g)+1\right)$ then

updateRoutingTable( $\mathrm{n} \mathrm{msg}$ )

In QOS-RCBRP, topology finding procedure in II-Algorithm is initiated by boundary nodes. Thus it is defined first with boundary node. A node can sense the presence of its neighbors when they relay ALIVE messages and know their hop distance to the cluster head from the ALIVE messages they relayed. Then a node can decide whether itself is a boundary node. Boundary nodes periodically initiates the topology finding procedure and send out EXAMINE messages. The EXAMINE message contains the local network topology of the boundary node. The EXAMINE message will be relayed back to the cluster head following the Rdft on each node along the shortest path back to the cluster head. Each intermediate node will add its local topology information to the EXAMINE message before relaying the message. In this way, each EXAMINE message contains the partial network topology along the path that it traversed and the cluster head will collect all the EXAMINE messages and construct the complete cluster topology. The combined cluster topology is then embedded in the next ALIVE message and sent out throughout the cluster. To enable inter-cluster routing, each cluster head periodically exchanges membership information using SUBSCRIBE message via its uplink. Then each cluster head will have knowledge about nodes in other clusters.
Cluster members can obtain the topology of the local cluster from the periodic ALIVE and populate their routing table using shortest path algorithms. The ALIVE message contains the snapshot of cluster topology captured during the last topology finding process. Still, this topology snapshot is not perfectly accurate as it only captures the topology in the past. The accuracy is determined by the frequency of topology finding and ALIVE emission. It is to be noted that this slight inaccuracy does not have significant impact on the routing performance. For destinations in other clusters, nodes will not find a valid route to it and these packets will be forwarded to the cluster head using the default route Rdft . Since the cluster head has the knowledge of members in other clusters, it can decide which cluster to forward to. Inter-cluster traffic are forwarded using the high capacity uplink directly to the destination cluster. The cluster head in the destination cluster will in turn forward the packets to destination. In addition, boundary nodes may also receive ALIVE messages from other clusters and they can establish direct and shorter route and forward the packets directly to the destination node across the cluster boundary. Each route entry is associated with a lifetime and it will be removed when expired. The data transferring algorithm is shown in III - Algorithm.

\section{II-Algorithm Topology Finding}

\author{
if isBoundaryNode(n) then \\ EXAMINE $=\operatorname{topology}(n)$ \\ Broadcast EXAMINE to Rdft(n) \\ if isClusterHead(n) then \\ updateTopology() \\ updateRoutingTable() \\ else \\ updateRoutingTable(n) \\ EXAMINE $=$ topology $($ EXAMINE $)+$ topology $(n)$ \\ Broadcast EXAMINE to Rdft(n)
}

\section{III-Algorithm Data Transferring}

if $\operatorname{dest}(p)==n$ then

receive $(p)$

else

if isClusterHead(n)then

NextHop $=$ searchRoutingTable $(\operatorname{dest}(p))$

if NextHop - NULL then

forward(p,NextHop)

else

$\operatorname{drop}(p)$

else

NextHop $=$ searchRoutingTable $(\operatorname{dest}(p))$

if NextHop - NULL then

forward ( $p$,NextHop)

else

$\operatorname{forward}(p, \operatorname{Rdft}(n))$

\section{SIMULATION SETTINGS AND PERFORMANCE METRICS}

Network Simulator 2 (NS2) is used to simulate QoS-RCBRP and AODV protocol; 50 to 250 mobile nodes starting from IP address 192.168 .1 .1 to 192.168 .1 .250 move in a $2000 \times 2000$ meter rectangular region for 200 seconds simulation time. The channel capacity of mobile nodes is set to the value ranging between 0.5 to 2 Mbps. We use the distributed coordination function (DCF) of IEEE 802.11 for wireless LANs. It has the functionality to notify the network layer about link breakage. We assume each node moves independently with the different mobility speed between $0.5 \mathrm{~m} / \mathrm{s}$ to $3 \mathrm{~m} / \mathrm{s}$. All nodes have the 
different transmission range ranging between 150 to 250 meters. The simulated traffic is Variable Bit Rate (VBR) with varying initial energy between 1.75 to 2.5 joules. The simulation settings are also represented in tabular format as shown in Table 1.

Table 1. NS2 Simulation Settings

\begin{tabular}{|l|l|}
\hline No. of Nodes & $50,100,150,200,250$ \\
\hline Terrain Size & 2000 X $2000 \mathrm{~m}$ \\
\hline MAC & $802.11 \mathrm{~b}$ \\
\hline Radio Transmission Range & 150 to 250 meters \\
\hline Simulation Time & 200 seconds \\
\hline Traffic Source & $\begin{array}{l}\text { VBR (Variable Bit } \\
\text { Rate) }\end{array}$ \\
\hline Packet Size & 512 KB \\
\hline Mobility Model & $\begin{array}{l}\text { Random Waypoint } \\
\text { Model }\end{array}$ \\
\hline Speed & 0.5 m/s to 3 m/s \\
\hline
\end{tabular}

\subsection{Performance Metrics}

The metrics are taken into account for comparing performance of the proposed QoS-RCBRP and AODV routing protocols. The metrics for reliability is extensively simulated using NS2. For ensuring reliability, the metrics such as delay, packet delivery ratio and throughput metrics are taken.

\section{RESULTS AND DISCUSSIONS}

From Fig. 3, delay metric it can see that the proposed QoSRCBRP has reduced delay than that of AODV. Fig.1 shows that the throughput is more in QoS-RCBRP compared to AODV. In Fig. 2, it can be see that, delivery ratio is higher than that of AODV routing protocol.

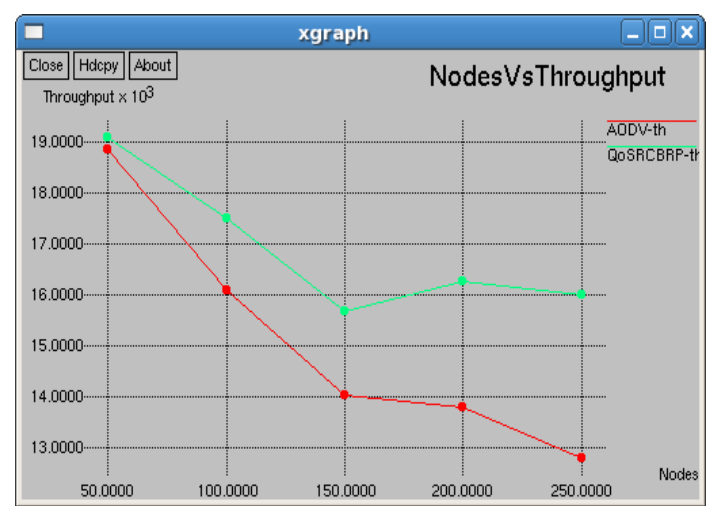

Fig.1 No. of Nodes Vs Throughput

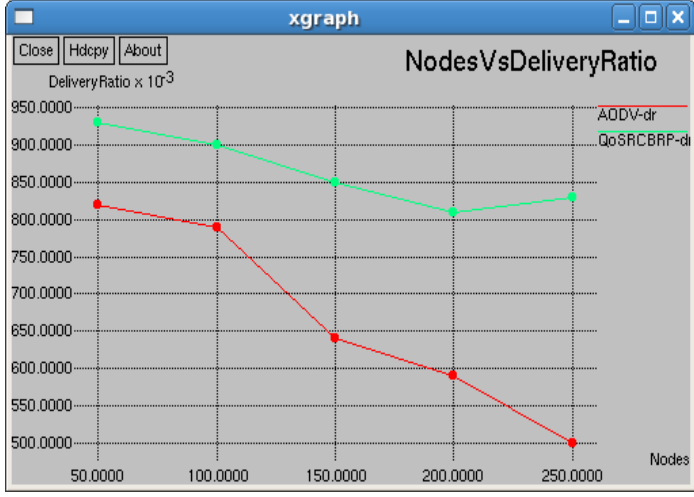

Fig.2 No. of Nodes Vs Delivery Ratio

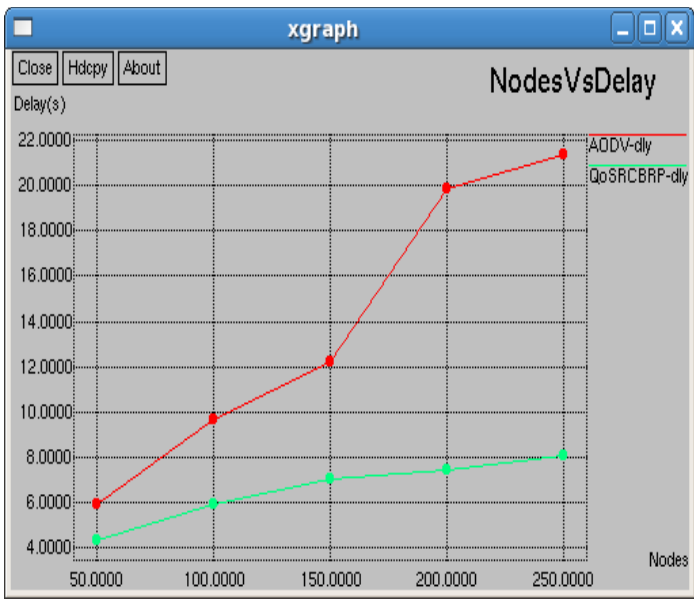

Fig.3 No. of Nodes Vs Delay

\section{CONCLUSIONS}

This research work delivered an efficient design and develops QoS aware reliable cluster based routing protocol (QoSRCBRP) for heterogeneous mobile ad hoc networks. The proposed routing protocol QoS-RCBRP makes use of mobile backbone to lessen the impact of node mobility. The proposed routing mechanism gets rid of the delay caused by cluster head selection and starts the mobile nodes to initialize the communication instantly after joining the cluster. The QoS metrics packet delivery ratio, delay and throughput are taken into account for comparing the proposed QoS-RCBRP with Ad-hoc On-demand Distance Vector (AODV) routing protocol. From dense simulation results it has proved that the proposed protocol attains better performance in terms of reduced delay, increased throughput and packet delivery ratio.

\section{ACKNOWLEDGEMENTS}

The first author thanks the management of Dr.N.G.P. Educational Institutions for providing career opportunity as an Assistant Professor in the Department of Computer Technology at Dr. N.G,P. Arts and Science College, Coimbatore. The second author thanks UGC for allocating Minor Research Project grant during Feb 2011 to Feb 2013 Grant Number 39-947/2010 (SR).

\section{REFERENCES}

[1] A. Zemlianov, G. de Veciana, Capacity of ad hoc wireless networks with infrastructure support, IEEE Journal on Selected Areas in Communications 23 (2005) 657-667. 
[2] T. Clausen, P. Jacquet, Optimized Link State Routing Protocol (OLSR), RFC 3626 (Experimental), 2003.

[3] C.E. Perkins, P. Bhagwat, Highly dynamic destinationsequenced distancevector routing (dsdv) for mobile computers, SIGCOMM Comput. Commun. Rev. 24 (1994) 234-244.

[4] X. Hong, K. Xu, M. Gerla, Scalable routing protocols for mobile ad hoc networks, IEEE Network 16 (2002) 1121.

[5] F. Theoleyre, F. Valois, Virtual structure routing in ad hoc networks, in: Communications, 2005. ICC 2005. 2005 IEEE International Conference on, vol. 5, 2005, pp. 3078-3082.

[6] X. Niu, Z. Tao, G. Wu, C. Huang, L. Cui, Hybrid cluster routing: An efficient routing protocol for mobile ad hoc networks, in: Communications, 2006. ICC '06. IEEE International Conference on, vol. 8, 2006, pp. 35543559 .

[7] Y. Ge, L. Lamont, L. Villasenor, Hierarchical olsr - a scalable proactive routing protocol for heterogeneous ad hoc networks, in: Wireless And Mobile Computing, Networking And Communications, 2005. (WiMob'2005), IEEE International Conference on, vol. 3, 2005, pp. 17-23.

[8] L. Villasenor-Gonzalez, Y. Ge, L. Lament, Holsr: a hierarchical proactive routing mechanism for mobile ad hoc networks, IEEE Communications Magazine 43 (2005) 118-125.

[9] T. Rasheed, K.A. Agha, U. Javaid, L. Reynaud, Clusterquality based hybrid routing for large scale mobile multihop networks, in: Wireless Communications and Networking Conference, 2007.WCNC 2007. IEEE, 2007, pp. 3052-3057.

[10] K. Xu, X. Hong, M. Gerla, An ad hoc network with mobile backbones, in: Communications, 2002. ICC 2002. IEEE International Conference on, vol. 5, 2002, pp. $3138-3143$

[11] K. Xu, M. Gerla, A heterogeneous routing protocol based on a new stable clustering scheme, in: MILCOM 2002. Proceedings, vol. 2, 2002, pp. 838-843.

[12] A. Iwata, C.-C. Chiang, G. Pei, M. Gerla, T.-W. Chen, Scalable routing strategies for ad hoc wireless networks, IEEE Journal on Selected Areas in Communications 17 (1999) 1369-1379.

[13] C. Lin, M. Gerla, Adaptive clustering for mobile wireless networks, IEEE Journal on Selected Areas in Communications 15 (1997) 1265-1275.

[14] M. Gerla, J. Tsai, Multicluster, mobile, multimedia radio network, in: Wireless Netw 1, 1995. Highest Connectivity Clustering.

[15] C. Chiang, H. Wu, W. Liu, Routing in clustered multihop, mobile wireless networks with fading channel, in: Proceedings of IEEE SICON, 1997. Least Cluster Change (LCC).

[16] D. Gavalas, G. Pantziou, C. Konstantopoulos, B. Mamalis, Clustering of mobile ad hoc networks: an adaptive broadcast period approach, in: Communications, 2006. ICC '06. IEEE International Conference on, vol. 9, 2006, pp. 4034-4039.

[17] B. Latre, J. Hocbeke, L. Peters, T.V. Leeuwen, I. Moerman, B. Dhoedt, P. Demeester, A heterogeneity based clustering heuristic for mobile ad hoc networks, in: Communications, 2004, IEEE International Conference on, vol. 6, 2004, pp. 3728-3733.

[18] A. Amis, R. Prakash, T. Vuong, D. Huynh, Max-min dcluster formation in wireless ad hoc networks, in: INFOCOM 2000. Nineteenth Annual Joint Conference of the IEEE Computer and Communications Societies. Proceedings. IEEE, vol. 1, 2000, pp. 32-41.

[19] G. Chen, F. Nocetti, J. Gonzalez, I. Stojmenovic, Connectivity based k-hop clustering in wireless networks, in: System Sciences, 2002. HICSS. Proceedings of the 35th Annual Hawaii International Conference on, 2002, pp. 2450-2459.

[20] S. Leng, Y. Zhang, H.-H. Chen, L. Zhang, K. Liu, A novel k-hop compound metric based clustering scheme for ad hoc wireless networks, in: Wireless Communications, IEEE Transactions on, vol. 8, 2009 pp. 367-375.

[21] X. Ma, A K-hop cluster maintaining mechanism for mobile ad hoc networks, in: Wireless Communications, Networking and Mobile Computing (WiCOM), 2011 7th International Conference on, pp. 1-4.

[22] M. Amjad, A. Zaman, K. Sakib, Efficient scalable clustering scheme for pseudolinear mobile ad hoc network, in: Wireless Communications, Networking and Mobile Computing (WiCOM), 2011 7th International Conference on, pp. 1-4.

[23] E. Dror, C. Avin, Z. Lotker, Fast randomized algorithm for hierarchical clustering in vehicular ad-hoc networks, in: Ad Hoc Networking Workshop (Med-Hoc-Net), 2011 The 10th IFIP Annual Mediterranean, 2011, pp. 1-8.

[24] I. Er, W. Seah, Mobility-based d-hop clustering algorithm for mobile ad hoc networks, in: Wireless Communications and Networking Conference, 2004 WCNC. 2004 IEEE, vol. 4, 2004, pp. 2359-2364.

[25] E. Sakhaee, A. Jamalipour, Stable clustering and communications in pseudolinear highly mobile ad hoc networks, in: Vehicular Technology, IEEE Transactions on, vol. 57, 2008, pp. 3769-3777.

[26] H. Cheng, J. Cao, X. Wang, S. Das, S. Yang, Stability aware multimetric clustering in mobile ad hoc networks with group mobility, in: Wirel. Commun. Mob. Comput., vol. 9, 2009, pp. 759-771. 
[27] C. Shea, B. Hassanabadi, S. Valaee, Mobility-based clustering in vanets using affinity propagation, in: Global Telecommunications Conference, 2009. GLOBECOM 2009. IEEE, 2009, pp. 1-6.

[28] C. Konstantopoulos, D. Gavalas, G. Pantziou, Clustering in mobile ad hoc networks through neighborhood stability-based mobility prediction, Computer Networks: The International Journal of Computer and Telecommunications Networking 52 (2008).

[29] M. Ni, Z. Zhong, H. Wu, D. Zhao, A new stable clustering scheme for highly mobile ad hoc networks, Wireless Communications and Networking Conference (WCNC), 2010 IEEE ( 2010) 1-6.

[30] E. Souza, I. Nikolaidis, P. Gburzynski, A new aggregate local mobility (alm) clustering algorithm for vanets, in: Communications (ICC), 2010 IEEE International Conference on, 2010, pp. 1-5.

[31] Y. Zhang, J.M. Ng, A distributed group mobility adaptive clustering algorithm for mobile ad hoc networks, in: Communications, 2008. ICC '08. IEEE International Conference on, 2008, pp. 3161-3165.
[32] C. Perkins, E. Belding-Royer, S. Das, Ad hoc OnDemand Distance Vector (AODV) Routing, RFC 3561 (Experimental), 2003.

[33] P. Jacquet, A. Laouiti, P. Minet, L. Viennot, Performance analysis of OLSR multipoint relay flooding in two ad hoc wireless network models, RSRCP Special issue on Mobility and Internet (2001).

[34] M. Ni, Z. Zhong, D. Zhao, MPBC: A mobility prediction-based clustering scheme for ad hoc networks, IEEE Transactions on Vehicular Technology 60 (2011) $4549-4559$.

[35] N. Maslekar, M. Boussedjra, J. Mouzna, H. Labiod, A stable clustering algorithm for efficiency applications in VANETs, in: Wireless Communications and Mobile Computing Conference (IWCMC), 2011 7th International, pp. 1188-1193. 